\title{
Existence of ruled wrappings in hyperbolic 3-manifolds
}

\author{
TERUHIKO SOMA
}

\begin{abstract}
We present a short elementary proof of an existence theorem of certain CAT $(-1)-$ surfaces in open hyperbolic 3-manifolds. The main construction lemma in Calegari and Gabai's proof of Marden's Tameness Conjecture can be replaced by an applicable version of our theorem. Finally, we will give a short proof of the conjecture along their ideas.
\end{abstract}

57M50; 30F40

Agol [1] and Calegari and Gabai [5] proved independently that any hyperbolic 3manifold $M$ with finitely generated fundamental group is homeomorphic to the interior of a compact 3-manifold. This is the affirmative answer to Marden's Tameness Conjecture in [8]. Subsequently, Choi [7] gave another proof of the conjecture, similar to Agol's, in the case when $M$ has no parabolic cusps.

We are here interested in arguments in [5], where the notion of "shrinkwrapping" was introduced. Shrinkwrappings play an important role in their proof. For the proof of the existence of shrinkwrappings and that of their CAT $(-1)$-property, Calegari and Gabai used very deep and rarefied arguments, which some readers, including the author, may find difficult to approach. This paper is intended to give an elementary proof of part of their proof by using ruled wrappings instead of shrinkwrappings.

For simplicity, we only consider the case when a hyperbolic 3-manifold has no parabolic cusps and will prove the following theorem.

Theorem 0.1 Let $N$ be an orientable hyperbolic 3-manifold without parabolic cusps, $\Delta$ a disjoint union of finitely many simple closed geodesics in $N$, and $f: \Sigma \longrightarrow N$ a 2 -incompressible map rel. $\Delta$ from a closed orientable surface $\Sigma$ of genus greater than 1 to $N \backslash \Delta$. Then there exists a homotopy $F: \Sigma \times[0,1] \longrightarrow N$ satisfying the following conditions.

(i) $F(x, 0)=f(x)$ for any $x \in \Sigma$.

(ii) $F(\Sigma \times[0,1)) \cap \Delta=\varnothing$.

(iii) The map $g: \Sigma \longrightarrow N$ defined by $g(x)=F(x, 1)$ is a CAT $(-1)-$ piecewise ruled map. 
Here, a continuous map $f: \Sigma \longrightarrow N$ is said to be 2-incompressible in $N$ rel. $\Delta$ if $f(\Sigma) \cap \Delta=\varnothing, f_{*}: \pi_{1}(\Sigma) \longrightarrow \pi_{1}(N \backslash \Delta)$ is injective, and for any simple noncontractible loop $l$ in $\Sigma$ the restriction $\left.f\right|_{l}$ is not freely homotopic in $N \backslash \Delta$ to a (multiplied) meridian of any component of $\Delta$. See Definition 1.4 for the definition of piecewise ruled maps. We say that a map $g$ satisfying properties (i)-(iii) as above or its image $g(\Sigma)$ is a CAT $(-1)$-ruled wrapping of $\Delta$ in $N$ homotopic to $f$. In fact, Theorem 0.1 is a special case of Proposition 2.1, which corresponds to the main construction lemma in [5].

In Section 4, we will give a short proof of Marden's Conjecture along ideas in [5]. Our proof is self-contained in the sense that it does not rely on published partial solutions to the conjecture. It is not hard to see that all arguments and proofs in this paper work also in the case when the ambient manifold $N$ has pinched negative curvature and hyperbolic cusps by invoking results in Canary [6, Section 4].

Acknowledgments The author would like to thank Brian Bowditch and the referee for helpful comments and suggestions.

\section{Completion of certain hyperbolic metrics}

For a closed subset $A$ in a metric space $(X, d)$, the $r$-neighborhood of $A$ in $X$, $\{y \in X ; d(y, A) \leq r\}$, is denoted by $\mathcal{N}_{r}(A)$ (or more strictly by $\mathcal{N}_{r}(A, X)$ ). In the case when $A$ is a single point set $\{x\}$, we also set $\mathcal{N}_{r}(\{x\})=\mathcal{B}_{r}(x)$. The link of $x$ in $X$ with radius $r,\{y \in X ; d(y, x)=r\}$, is denoted by $\mathcal{S}_{r}(x)$.

Let $U$ be a simply connected incomplete hyperbolic 3-manifold with metric completion $\bar{U}$ such that each component $l$ of $L=\bar{U} \backslash U$ is a geodesic line, and there exists a constant $c>0$ with $\operatorname{dist}(x, y) \geq 3 c$ for any points $x, y$ contained in distinct components of $L$. Moreover, we suppose that for any component $l$ of $L$ there exists an infinite cyclic branched covering $p_{l}: \mathcal{N}_{c}(l, \bar{U}) \longrightarrow \mathcal{N}_{c}\left(j, \mathbf{H}^{3}\right)$ branched over a geodesic line $j$ of $\mathbf{H}^{3}$, such that the restriction $\left.p_{l}\right|_{\mathcal{N}_{c}(l, \bar{U}) \backslash l}$ is a locally isometric covering. From the definition, $\mathcal{N}_{c}(l, \bar{U})$ is homeomorphic to the quotient space of $\mathbf{R}^{2} \times[0,1]$ by the identification map $a: \mathbf{R}^{2} \times\{0\} \longrightarrow \mathbf{R}$ defined by $a(x, y, 0)=x$.

Suppose that $\sigma$ is a shortest arc in $\bar{U}$ connecting two given points and consisting of two hyperbolic segments $\sigma_{1}, \sigma_{2}$ with $\sigma \cap L=\sigma_{1} \cap \sigma_{2}=\{x\}$. Let $x_{i}(i=1,2)$ be the point in $\sigma_{i}$ with $\operatorname{dist}\left(x_{1}, l\right)=\operatorname{dist}\left(x_{2}, l\right)=s>0$, where $l$ is the component of $L$ containing $x$; see Figure 1 (a). There exist totally geodesic half planes $P_{i}$ in $\bar{U}$ with $\sigma_{i} \subset P_{i}$ and $\partial P_{i}=l$. Since the subsegment $\tau$ of $\sigma$ with $\partial \tau=\left\{x_{1}, x_{2}\right\}$ is the shortest 


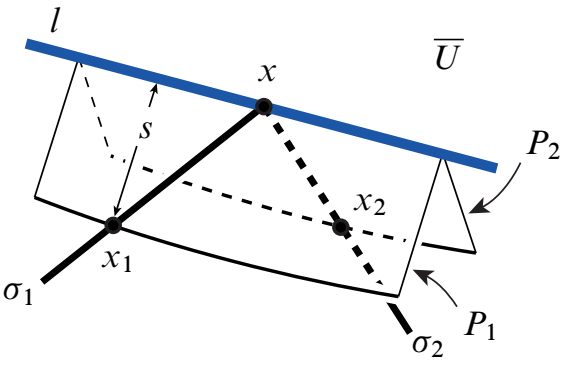

(a)

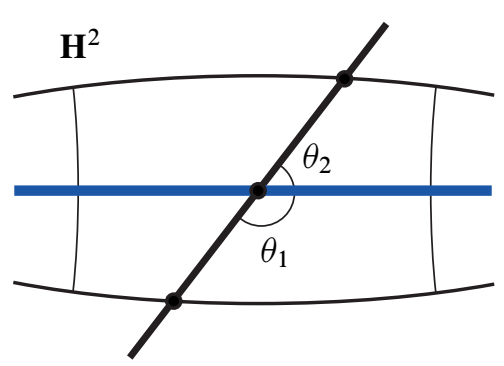

(b)

Figure 1

$\operatorname{arc}$ in $\bar{U}$ connecting $x_{1}$ with $x_{2}$, we have

$$
\theta_{1}+\theta_{2}=\pi
$$

where $\theta_{i}$ is the angle made by $\sigma_{i}$ and a fixed ray in $l$ emanating from $x$. This fact is easily seen by considering the developing of $P_{1} \cup P_{2}$ on $\mathbf{H}^{2}$; see Figure 1 (b).

For any $d$ with $0<d \leq c$, the set $\mathcal{B}_{d}(x, \bar{U})$ is homeomorphic to the subset of $\mathbf{R}^{3}$

$$
\left\{(u, v, w) \in \mathbf{R}^{3} ; u^{2}+v^{2}+w^{2} \leq 1, w>0\right\} \cup\left\{(u, 0,0) \in \mathbf{R}^{3} ;-1 \leq u \leq 1\right\} .
$$

In particular, $\mathcal{B}_{d}(x, \bar{U})$ is simply connected. The image $p_{l}\left(\mathcal{B}_{d}(x, \bar{U})\right)$ coincides with the hyperbolic ball $\mathcal{B}_{d}\left(\hat{x}, \mathbf{H}^{3}\right)$, where $\hat{x}=p_{l}(x)$. Rescaling the metric on the boundary $S=\mathcal{S}_{d}\left(\hat{x}, \mathbf{H}^{3}\right)$ of the ball, we have the spherical metric $v$ on $S$ isometric to the unit sphere in the Euclidean 3-space. Consider the metric on $\widetilde{S}=\mathcal{S}_{d}(x, \bar{U})$, still denoted by $v$, so that the infinite cyclic branched covering $p_{l} \mid \widetilde{S}: \widetilde{S} \longrightarrow S$ is locally pathwise isometric. Here, $p_{l} \mid \widetilde{S}$ being locally pathwise isometric means that length $_{v}(\alpha)=$ length $_{v}\left(p_{l}(\alpha)\right)$ for any rectifiable arc $\alpha$ in $\widetilde{S}$. One can take $d>0$ so that $\sigma^{\prime}=\sigma \cap \mathcal{B}_{d}(x)$ is a geodesic segment in $\mathcal{B}_{d}(x)$ with $\partial \sigma^{\prime} \subset \widetilde{S}$. Let $\gamma$ be any rectifiable arc in $\widetilde{S}$ with $\partial \gamma=\partial \sigma^{\prime}$. Since $\mathcal{B}_{d}(x)$ is simply connected, $\gamma$ is homotopic rel. $\partial \gamma$ to $\sigma^{\prime}$ in $\mathcal{B}_{d}(x)$. Then the following lemma is proved immediately from the equality (1-1) and by checking the situation of $\hat{\gamma}=p_{l}(\gamma)$ in $S$; see Figure 2 .

Lemma 1.1 length $_{v}(\gamma) \geq \pi$.

For any $k \leq 0$, let $\mathbf{F}(k)$ be a complete Riemannian plane of constant curvature $k$. A simply connected geodesic metric space $X$ is called a CAT $(k)$-space if any geodesic triangle $\Delta$ in $X$ is not thicker than a comparison triangle $\bar{\Delta}$ in $\mathbf{F}(k)$, that is, for any two points $s$ and $t$ in the edges of $\Delta$ and their comparison points $\bar{s}$ and $\bar{t}$ in $\bar{\Delta}$, $\operatorname{dist}_{X}(s, t) \leq \operatorname{dist}_{\mathbf{F}(k)}(\bar{s}, \bar{t})$. See Bridson-Haefliger [3] for fundamental properties of such spaces. 


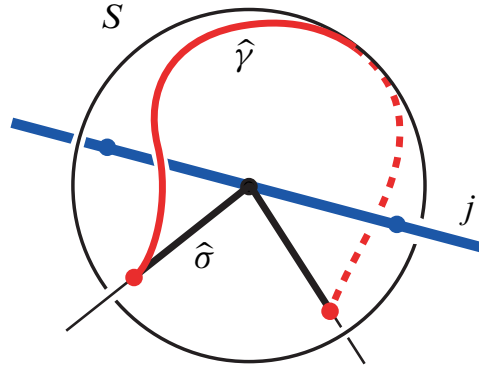

(a)

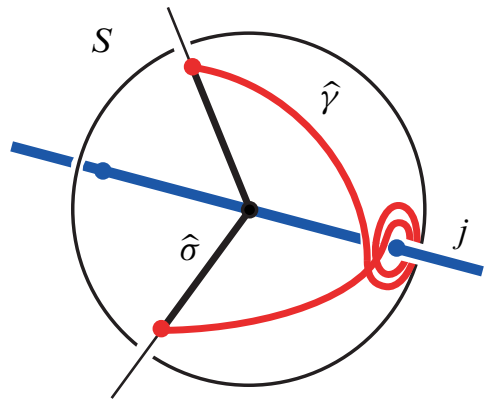

(b)

Figure 2: $j=p_{l}(l), \hat{\sigma}=p_{l}\left(\sigma^{\prime}\right)$. (b) is the case when $\hat{\gamma}$ winds around $j$ more than once, but length ${ }_{v}(\gamma)=$ length $_{v}(\hat{\gamma})$ does not exceed $\pi$ very much.

Lemma 1.2 $\bar{U}$ is a CAT(-1)-space.

Proof By the generalized Cartan-Hadamard Theorem [3, Chapter II.4, Theorem 4.1(2)], it suffices to show that, for any point $x \in \bar{U}$, there exists $r>0$ such that $B_{r}(x)$ is a CAT $(-1)$-space. If $x \in U$ then $B_{r}(x)$ is obviously a CAT(-1)-space if we take $r$ so that $B_{r}(x) \cap L=\varnothing$. So suppose that $x \in L$. Let $\Delta$ be any triangle in $B_{c}(x)$ with geodesic edges $\gamma_{1}, \gamma_{2}, \gamma_{3}$. Each Int $\gamma_{i}$ either meets $L$ at most one point $p_{i}$ or is contained in $L$. From this, we know that the union $\gamma_{1} \cup \gamma_{2} \cup \gamma_{2}$ bounds a triangle $\Delta_{0}$ in $B_{c}(x)$ consisting of at most three totally geodesic hyperbolic subtriangles. In fact, when $p_{1}, p_{2}, p_{3}$ exist, the totally geodesic triangle with vertices $p_{1}, p_{2}, p_{3}$ is degenerated to a geodesic segment in $L$. By Lemma 1.1, the internal angle of $\Delta_{0}$ at any $p_{i}$ is at least $\pi$. This shows that $\Delta_{0}$ is not thicker than a comparison triangle in $\mathbf{H}^{2}$. It follows that $B_{c}(x)$ is a $\operatorname{CAT}(-1)$-space.

It is not hard to see that any geodesic segment in $\bar{U}$ is a broken line consisting of finitely many hyperbolic segments, and any vertex of the broken line other than its end points lies in $L$. By Lemma 1.2, a geodesic segment $\sigma$ in $\bar{U}$ connecting two given points is uniquely determined. Moreover, $\sigma$ varies continuously with its end points.

Let $Z$ be an incomplete hyperbolic 3-manifold such that the total space of the universal covering $q: U \longrightarrow Z$ has the induced metric as above. We suppose moreover that for the metric completion $\bar{Z}$ each component $l$ of $\bar{Z} \backslash Z$ is either a geodesic line or a geodesic loop. That is, no component of $\bar{Z} \backslash Z$ is a one point set. Then $q: U \longrightarrow Z$ is extended to a locally pathwise isometric map $\bar{q}: \bar{U} \longrightarrow \bar{Z}$. Note that the frontier $\{x \in \bar{Z} ; \operatorname{dist}(x, l)=c\}$ of $\mathcal{N}_{c}(l, \bar{Z})$ in $\bar{Z}$ is homeomorphic to either $\mathbf{R}^{2}$ or an open annulus or a torus. 
Remark 1.3 Even in the case when $\mathcal{N}_{c}(l, \bar{Z})$ is homeomorphic to a solid torus, we always suppose that homotopies in $\bar{Z}$ starting from a continuous map $f: A \longrightarrow Z$ never cross $l$ (possibly they touch $l$ ), where $A$ is a manifold of dimension less than three. In other words, we only consider homotopies $F: A \times[0,1] \longrightarrow \bar{Z}$ which can be covered by a map $\widetilde{F}: \widetilde{A} \times[0,1] \longrightarrow \bar{U}$, where $\tilde{A}$ is the universal covering space of $A$.

Definition 1.4 Let $f: \Sigma \longrightarrow \bar{Z}$ be a continuous map from a closed orientable surface $\Sigma$. Suppose that $\Sigma$ admits a cell decomposition $K$ consisting of finitely many triangular 2 -cells. We say that $f$ is a piecewise ruled map with respect to $K$ if (i) for each edge of $e$ of $K, f(e)$ is a broken line consisting of finitely many hyperbolic segments, and (ii) for each 2-cell $F$ of $K, f(F)$ is a ruled triangle based on a single vertex. Since the Gaussian curvature at any smooth point of a ruled surface is nonpositive, the (intrinsic) curvature of $\Sigma$ at any smooth point is at most -1 . This map $f$ is called a CAT(-1)-piecewise ruled map if the cone-angle of $\Sigma$ at any singular point is at least $2 \pi$.

\section{Applicable version of Theorem 0.1}

Throughout this section, we assume that all hyperbolic 3-manifolds and surfaces are orientable.

Let $N$ be a complete hyperbolic 3-manifold $N$ without parabolic cusps, and $W$ a 3 -dimensional compact connected submanifold of $N$. Consider a link $\Delta$ in Int $W$ consisting of finitely many disjoint simple closed geodesics in $N$. Let $p: X \longrightarrow W$ be the covering of $W$ associated to a finitely generated subgroup of $\pi_{1}(W)$. Here we make the following assumptions, which correspond to those in the main construction lemma [5, Lemma 2.3].

(i) $\partial W$ is incompressible in $N \backslash \Delta$.

(ii) There exists a union $\widehat{\Delta}$ of components of $p^{-1}(\Delta)$ such that the restriction $p \mid \widehat{\Delta}: \widehat{\Delta} \longrightarrow \Delta$ is a homeomorphism.

(iii) There exists a continuous map $f: \Sigma \longrightarrow X$ from a closed surface $\Sigma$ of genus $m>1$ which is 2 -incompressible in $X$ rel. $\widehat{\Delta}$.

Set $X^{\circ}=X \backslash p^{-1}(\Delta)$. The fundamental group $\pi_{1}\left(X^{\circ}\right)$ may be infinitely generated. By (i), the restriction $p^{\circ}=p \mid X^{\circ}: X^{\circ} \longrightarrow W \backslash \Delta \subset N \backslash \Delta$ is $\pi_{1}$-injective. Thus, we may assume that $X^{\circ}$ is a subset of the total space of the covering $q: Y^{\circ} \longrightarrow N \backslash \Delta$ associated to the subgroup $p_{*}^{\circ}\left(\pi_{1}\left(X^{\circ}\right)\right)$ of $\pi_{1}(N \backslash \Delta)$ and the inclusion $i: X^{\circ} \longrightarrow Y^{\circ}$ is a homotopy equivalence. Since $\partial X^{\circ}=\partial X=p^{-1}(\partial W)$ is a deformation retract of 
$Y^{\circ} \backslash$ Int $X^{\circ}$, condition (iii) implies that $f: \Sigma \longrightarrow Y$ is 2 -incompressible in $Y$ rel. $\widehat{\Delta}$, where $Y=X \cup\left(Y^{\circ} \backslash\right.$ Int $\left.X^{\circ}\right)=X \cup Y^{\circ}$. The complement $Z=Y \backslash \widehat{\Delta}$ has the induced incomplete metric as was studied in Section 1. Let $\bar{Z}$ be the metric completion of $Z$.

With the notation and assumptions as above, we will prove the following proposition.

Proposition 2.1 There exists a homotopy $F: \Sigma \times[0,1] \longrightarrow \bar{Z}$ which never crosses $\widehat{\Delta}$ and connects $f$ with a CAT(-1)-ruled wrapping $g: \Sigma \longrightarrow \bar{Z}$ of $\widehat{\Delta}$.

Proof Let $c_{1}, \ldots, c_{3 m-3}$ be mutually disjoint simple loops in $\Sigma$ which define a pants decomposition of $\Sigma$. Consider a cell decomposition $K$ of $\Sigma$ consisting of triangular 2-cells and such that each vertex of $K$ is contained in $c_{1} \cup \cdots \cup c_{3 m-3}$. If necessary by deforming $f$ by a homotopy in the sense of Remark 1.3 we may assume that each $f\left(c_{i}\right)$ is a closed geodesic in $\bar{Z}$, and $f(e)$ is a geodesic segment in $\bar{Z}$ for any edge $e$ of $K$ not contained in $c_{1} \cup \cdots \cup c_{3 m-3}$. In fact, $f\left(c_{i}\right)$ is the image of an axis of a hyperbolic transformation on the metric completion $\bar{U}$ of the universal covering space of $Z$. See for example [3, Theorem 6.8(1)]. For any 2-cell $F$ of $K$, take a vertex $v_{0}$ and the opposite edge $e_{0}$. Then, $f \mid F$ can be homotoped rel. $\partial F$ to a map $g \mid F$ such that $g(F)$ is a ruled triangle consisting of all geodesic segments connecting $f\left(v_{0}\right)$ with points of $f\left(e_{0}\right)$. These $g \mid F$ define a piecewise ruled map $g: \Sigma \longrightarrow \bar{Z}$ homotopic to $f$. From our construction of $g$, for any singular point $g(v)$ of $g(\Sigma)$, there exists an $\operatorname{arc} \alpha$ in $\Sigma$ with Int $\alpha \ni v$ and such that $g(\alpha)$ is a geodesic segment in $\bar{Z}$. If $g(v)$ is not an element of $\bar{Z} \backslash Z$, then it is easily seen that the cone-angle of $\Sigma$ at $v$ is at least $2 \pi$. So we may assume that $g(v)$ is contained in a component $l$ of $\bar{Z} \backslash Z$. For any sufficiently small $d>0, \alpha$ divides the circle $\mathcal{S}_{d}(v, \Sigma)$ into two arcs $\gamma_{1}, \gamma_{2}$. By Lemma 1.1, the $v$-length of $g\left(\gamma_{i}\right)(i=1,2)$ in $\mathcal{S}_{d}(g(v), \bar{Z})$ is at least $\pi$. Thus, the cone-angle of $\Sigma$ at $v$ is at least $2 \pi$. This shows that $g$ is a CAT(-1)-ruled wrapping of $\widehat{\Delta}$ in $\bar{Z}$.

Note that Theorem 0.1 is proved quite similarly to Proposition 2.1 by considering $(N, \Delta)$ instead of $(\bar{Z}, \widehat{\Delta})$.

\section{Compact cores and end reductions}

A 3-manifold $X$ is topologically tame if there exists an embedding $f: X \longrightarrow Y$ into a compact manifold $Y$ with $f(X) \supset$ Int $Y$. Throughout this section, we suppose that $M$ is an orientable, open, irreducible and connected 3-manifold with finitely generated fundamental group. An end $\mathcal{E}$ of $M$ is said to be topologically tame if there exits a closed neighborhood of $\mathcal{E}$ in $M$ homeomorphic to $S \times[0, \infty)$ for some closed 
connected surface $S$. It is easily seen that the open 3-manifold $M$ is topologically tame if and only if each end of $M$ is so.

Scott [10] proved that $M$ contains a 3-dimensional submanifold $C$, called a compact core of $M$, such that the inclusion $i: C \longrightarrow M$ is a homotopy equivalence. Let $S$ be the component of $\partial C$ facing an end $\mathcal{E}$ of $M$, and $p: \tilde{M} \longrightarrow M$ the covering associated with the image of $\pi_{1}(S)$ in $\pi_{1}(M)$. There exists a compact core $\widetilde{C}$ of $\widetilde{M}$ such that $\partial \widetilde{C}$ has a component $\widetilde{S}$ mapped onto $S$ homeomorphically by $p$. The manifold $\widetilde{C}$ is a compression body, ie, it is homeomorphic to $E \cup h_{1} \cup \cdots \cup h_{m}$ where $E$ is either a 3-ball or $F \times[0,1]$ for some closed surface $F$ consisting of nonspherical components and the $h_{i}$ 's are 1-handles attached to one side of $E$. In particular, when $E$ is a 3 -ball, the compression body $\widetilde{C}$ is a handlebody. Note that the end $\widetilde{\mathcal{E}}$ of $\tilde{M}$ faced by $\widetilde{S}$ is topologically tame if and only if $\mathcal{E}$ is also.

Let $\Delta=\delta_{1} \cup \cdots \cup \delta_{i_{0}}$ be an $i_{0}$-component link in the compression body $\widetilde{C}$ such that $\left[\delta_{k}\right]\left(k=1, \cdots, i_{0}-1\right)$ form a basis for the free abelian group $H_{1}(\widetilde{C}, \mathbf{Z})$ and $\left[\delta_{i_{0}}\right]=\left[\delta_{1}\right]+\cdots+\left[\delta_{i_{0}-1}\right]$. An advantage of considering compression bodies is that any nontrivial free decomposition of $\pi_{1}(\widetilde{C})$ induces a nontrivial decomposition of $H_{1}(\widetilde{C} ; \mathbf{Z})$. In particular, this implies that the link $\Delta$ is algebraically disk-busting, that is, for any nontrivial free decomposition $A * B$ of $\pi_{1}(\widetilde{C})$, there exists a component $\delta_{k}$ of $\Delta$ such that the element of $\pi_{1}(\tilde{C})$ represented by $\delta_{k}$ is neither conjugate into $A$ nor $B$.

Some results in Myers [9] concerning end reductions play an important role in the proof of Theorem 4.1. The paper is useful also as an expository article on end reductions. A compact, connected, 3-dimensional submanifold $R$ of $M$ is regular if $M \backslash R$ is irreducible and the closure of any component of $M \backslash R$ in $M$ is not compact. Let $\Delta$ be a link in $M$ each component of which is noncontractible in $M$. An open submanifold $V$ of $M$ containing $\Delta$ is called an end reduction of $M$ at $\Delta$ if it satisfies the following conditions.

(i) No component of $M \backslash V$ is compact.

(ii) There exists a sequence $\left\{R_{n}\right\}$ of regular submanifolds of $M$ with $\Delta \subset R_{1}$, $R_{n} \subset$ Int $R_{n+1}, V=\bigcup_{n} R_{n}$ and such that $\partial R_{n}$ is incompressible in $M \backslash \Delta$.

(iii) $V$ satisfies the engulfing property at $\Delta$, that is, for any regular submanifold $N$ of $M$ with $\Delta \subset$ Int $N$ such that $\partial N$ is incompressible in $M \backslash \Delta, V$ is ambient isotopic rel. $\Delta$ to a manifold containing $N$.

We refer to Brin-Thickstun [4] for the existence and uniqueness up to isotopy of end reductions. According to Myers [9, Theorem 9.2], if the link $\Delta$ is algebraically disk-busting, then an end reduction $V$ of $M$ at $\Delta$ is connected and the homomorphism $i_{*}: \pi_{1}(V) \longrightarrow \pi_{1}(M)$ induced from the inclusion is an isomorphism. 


\section{Proof of Marden's Conjecture}

Our proof of Marden's Conjecture is based on that of Calegari-Gabai [5], but the importance of the disk-busting property is suggested by Agol [1]. For simplicity, we only consider hyperbolic 3-manifolds without parabolic cusps. It is not hard to modify our argument for the case of manifolds with parabolic cusps.

Theorem 4.1 (Marden's Tameness Conjecture) Let $N$ be an orientable hyperbolic 3 -manifold without parabolic cusps. If $\pi_{1}(N)$ is finitely generated, then $N$ is topologically tame.

First of all, we fix the setting for the proof. It suffices to show that each end $\mathcal{E}$ of $N$ is topologically tame. As was seen in Section 3, we may assume that a compact core $C$ of $N$ is a compression body. Let $S$ be the component of $\partial C$ facing $\mathcal{E}$. If $\mathcal{E}$ is geometrically finite, that is, $C$ is locally convex in $S$, then it is well known that $\mathcal{E}$ is topologically tame, for example see Marden [8]. So we may assume that $\mathcal{E}$ is not geometrically finite. Then Bonahon [2] shows that there exists a sequence $\left\{\delta_{i}\right\}$ of closed geodesics in $N$ exiting $\mathcal{E}$. If necessary, by adding finitely many closed geodesics to $\left\{\delta_{i}\right\}$ one can suppose that $\Delta_{i}=\delta_{1} \cup \cdots \cup \delta_{i}$ is algebraically disk-busting for all $i$ not less than some fixed integer $i_{0}>0$. If necessarily, by slightly deforming the hyperbolic metric in a small neighborhood of $\bigcup_{i} \delta_{i}$ in $N$ we may assume that the closed geodesics $\delta_{i}$ are simple and mutually disjoint, ie, each $\Delta_{i}$ is a link in $N$. In fact, the resulting metric is no longer hyperbolic but pinched negatively curved. However, all the results concerting hyperbolic manifolds which we need, eg Proposition 2.1 in Section 2, still hold under this metric if we replace $\operatorname{CAT}(-1)$ with $\operatorname{CAT}\left(-a^{2}\right)$, where $-a^{2}$ is the supremum of sectional curvatures of $N$ with respect to the new metric. We refer to Canary [6, Sections 4 and 5] for standard arguments on such a metric deformation.

For any $i \geq i_{0}$, let $V_{i}$ be an end reduction of $N$ at $\Delta_{i}$. By [9], the $\pi_{1}$-homomorphism induced from the inclusion $V_{i} \longrightarrow N$ is an isomorphism. It follows that a compact core $C_{i}$ of $V_{i}$ is also a compact core of $N$, and each $\delta_{k}(k=1, \ldots, i)$ is freely homotopic in $V_{i}$ to a loop of $C_{i}$. By property (ii) of the end reduction $V_{i}$, there exists a regular submanifold $W_{i}$ of $V_{i}$ containing both $C_{i}$ and the traces of these free homotopies in $C_{i}$ and such that $\partial W_{i}$ is incompressible in $N \backslash \Delta_{i}$. In fact, $R_{n}$ in Section 3 with sufficiently large $n$ satisfies the properties of $W_{i}$. Since the inclusion $C_{i} \longrightarrow W_{i} \longrightarrow N$ is $\pi_{1}$-isomorphic, $\pi_{1}\left(C_{i}\right)$ can be regarded as a subgroup of $\pi_{1}\left(W_{i}\right)$. Consider the covering $p_{i}: X_{i} \longrightarrow W_{i}$ associated to $\pi_{1}\left(C_{i}\right) \subset \pi_{1}\left(W_{i}\right)$. Let $\hat{\delta}_{k}$ be a component of $p_{i}^{-1}\left(\delta_{k}\right)$ such that $p_{i} \mid \widehat{\delta}_{k}: \widehat{\delta}_{k} \longrightarrow \delta_{k}$ is homeomorphic, and let $\widehat{\Delta}_{i}=\widehat{\delta}_{1} \cup \cdots \cup \hat{\delta}_{i}$. The 
component $S_{i}$ of $\partial C_{i}$ facing $\mathcal{E}$ has a lift $S_{i}^{\bullet}$ to $X_{i}$ which is also a boundary component of a compact core of $X_{i}$.

Lemma 4.2 $X_{i}$ is topologically tame.

Proof The claim in [5, page 426] shows that $W_{i}$ is an atoroidal manifold such that $\partial W_{i}$ has a component of genus greater than 1 . This fact together with Canary [6] proves that $X_{i}$ is topologically tame.

Here, we will give an another proof without invoking the atoroidality of $W_{i}$. First we outline the proof. Divide the covering $p_{i}: X_{i} \longrightarrow W_{i}$ to restricted coverings associated with a torus decomposition of $W_{i}$. By finite generation, all but finitely many restrictions are universal coverings. By a result in Waldhausen [14], the total spaces of such universal coverings are topologically tame. Other total spaces cover atoroidal Haken manifolds with non-torus boundary component, and hence they are topologically tame. Since $X_{i}$ is obtained by attaching topologically tame manifolds to each other along simply connected boundary components, Simon's Combination Theorem [11] shows that $X_{i}$ is also topologically tame.

More precisely, let $\mathcal{T}_{i}=T_{1} \cup \cdots \cup T_{m}$ be a maximal union of mutually disjoint and nonparallel incompressible tori in Int $W_{i}$. Since $W_{i}$ is regular and $N$ is atoroidal and irreducible, each $T_{j}$ bounds a compact manifold $A_{j}$ which is contained in a $3-$ ball in $N$ and homeomorphic to the exterior of a nontrivial knot in $S^{3}$. Either any two $A_{j}$ are mutually disjoint or one of them contains the other. Set $\mathcal{A}=A_{1} \cup \cdots \cup A_{m}$. Note that, for any component $\hat{A}$ of $p_{i}^{-1}(\mathcal{A})$, the image $\operatorname{inc}_{i} \circ p_{i}(\widehat{A})$ is contained in a 3-ball in $N$, where inc $_{i}: W_{i} \longrightarrow N$ is the inclusion. Since inc $_{i} \circ p_{i}: X_{i} \longrightarrow N$ is $\pi_{1}$-isomorphic, it follows that $\hat{A}$ is simply connected. Then $\hat{A}$ is topologically tame by [14, Theorem 8.1]. Since each component of $\partial \hat{A}$ is simply connected, $p_{i}^{-1}(\partial \mathcal{A})$ induces a free decomposition of $\pi_{1}\left(X_{i}\right)$. The classical Grushko Theorem implies that the fundamental group of any component $\hat{B}$ of $p_{i}^{-1}\left(W_{i} \backslash \operatorname{Int} \mathcal{A}\right)$ is finitely generated. Since $W_{i} \backslash \operatorname{Int} \mathcal{A}$ is an atoroidal Haken manifold such that one of the boundary components has genus greater than 1 , Int $\widehat{B}$ is topologically tame by [6, Proposition 3.2]. In the present case, it is not hard to show that $\widehat{B}$ is also topologically tame. See Soma [12] for a more general case. Finally, Theorem 3.1 in [11] implies that $X_{i}$ is topologically tame.

Proof of Theorem 4.1 By Lemma 4.2, $X_{i}$ is realized as the interior of a compact manifold $\bar{X}_{i}$. Let $\bar{S}_{i}$ be the component of $\partial \bar{X}_{i}$ facing $S_{i}^{\bullet}$ in $X_{i}$, and $\widehat{S}_{i}$ a closed surface in Int $X_{i}$ obtained by a small isotopy of $\bar{S}_{i}$ in $\bar{X}_{i}$. We show that $\hat{S}_{i}$ is 2incompressible in $X_{i}$ rel. $\widehat{\Delta}_{i}$. If not, there would exist a compressing disk $D$ for $\bar{S}_{i}$ in $\bar{X}_{i}$ such that the intersection $D \cap \widehat{\Delta}_{i}$ consists of at most one point. If $D$ separates 
$X_{i}$, then $\pi_{1}\left(X_{i}\right)$ has a nontrivial free decomposition $A * B$ each factor of which is isomorphic to the fundamental group of a component of $X_{i} \backslash D$. Otherwise, $\pi_{1}\left(X_{i}\right)$ is isomorphic to $A * \mathbf{Z}$ with $A=\pi_{1}\left(X_{i} \backslash D\right)$. When $D \cap \widehat{\Delta}_{i}=\varnothing$, any element of $\pi_{1}\left(X_{i}\right)$ represented by a component of $\widehat{\Delta}_{i}$ is conjugate into one of the factors. When $D \cap \widehat{\delta}_{j} \neq \varnothing$ for some component $\hat{\delta}_{j}$ of $\widehat{\Delta}_{i}$, one can suppose that the cyclic factor $\mathbf{Z}$ is generated by the element represented by $\hat{\delta}_{j}$. Any element represented by a component of $\widehat{\Delta}_{i} \backslash \widehat{\delta}_{j}$ is conjugate into $A$. It follows that $\widehat{\Delta}_{i}$ is not algebraically disk-busting in $X_{i}$. Since $\left(\text { inc }_{i} \circ p_{i}\right)_{*}: \pi_{1}\left(X_{i}\right) \longrightarrow \pi_{1}(N)$ is isomorphic and inc ${ }_{i} \circ p_{i}\left(\widehat{\Delta}_{i}\right)=\Delta_{i}$, the link $\Delta_{i}$ would not be algebraically disk-busting in $N$, a contradiction. One can show similarly that $\widehat{S}_{i}$ is 2-incompressible in $X_{i}$ also rel. $\widehat{\Delta}_{i ; i_{0}}=\widehat{\Delta}_{i} \cap p_{i}^{-1}\left(\Delta_{i_{0}}\right)$.

For any $i \geq i_{0}$, let $\bar{q}_{i}: \bar{Z}_{i} \longrightarrow N$ be the locally pathwise isometric map extending the covering $q_{i}: Y_{i}^{\circ} \longrightarrow N \backslash \Delta_{i}$ given in Section 2 which satisfies $q_{i}=p_{i}$ on $X_{i} \backslash p_{i}^{-1}\left(\Delta_{i}\right)$. Note that $\bar{Z}_{i}$ is the metric completion of $Z_{i}=X_{i} \cup Y_{i}^{\circ} \backslash \widehat{\Delta}_{i}$. By Proposition 2.1, $\widehat{S}_{i}$ is homotopic in $\bar{Z}_{i}$ to a $\operatorname{CAT}\left(-a^{2}\right)$-ruled wrapping $\widehat{\Sigma}_{i}$ without crossing $\widehat{\Delta}_{i}$. The image $\Sigma_{i}=\bar{q}_{i}\left(\hat{\Sigma}_{i}\right)$ is also a CAT $\left(-a^{2}\right)$-surface homotopic in $N$ to $S_{i}$.

Since the components $S_{i}$ of $\partial C_{i}$ are homeomorphic to each other, all $\hat{\Sigma}_{i}$ are closed surfaces of the same genus. Fix an $\varepsilon>0$ less than the Margulis constant for $N$ and so that $\mathcal{N}_{2 \varepsilon}\left(\Delta_{i_{0}}, N\right)$ is a tubular neighborhood of $\Delta_{i_{0}}$ in $N$. Let $l$ be any simple noncontractible loop in $\hat{\Sigma}_{i}$ of length less than $\varepsilon$. If $l$ were contractible in $\bar{Z}_{i}$, then $l$ would either bound a disk in $\bar{Z}_{i}$ disjoint from $\widehat{\Delta}_{i ; i_{0}}$ or be contained in $\mathcal{N}_{2 \varepsilon}\left(\widehat{\Delta}_{i ; i_{0}}, \bar{Z}_{i}\right) \backslash \widehat{\Delta}_{i ; i_{0}}$. In either case, this contradicts that $\hat{\Sigma}_{i}$ is 2 -incompressible rel. $\widehat{\Delta}_{i ; i_{0}}$. Thus $l$ is not contractible in $\bar{Z}_{i}$, and hence $\bar{q}_{i}(l)$ is contained in the $\varepsilon$-thin part $N_{\text {thin }(\varepsilon)}$ of $N$. From this fact together with Bounded Diameter Lemma [5, Lemma 1.15] for CAT $\left(-a^{2}\right)$-surfaces, we know that the diameter of any component of $\Sigma_{i} \backslash N_{\text {thin }}(\varepsilon)$ is less than a constant independent of $i$. Let $\widehat{\alpha}_{i}$ be a ray in $\bar{Z}_{i}$ beginning at $\hat{\delta}_{i}$ and covering a proper ray $\alpha_{i}$ in $N$ such that the sequence $\left\{\alpha_{i}\right\}$ exits $\mathcal{E}$. Since the algebraic intersection number of $\hat{\alpha}_{i}$ with $\hat{S}_{i}$ is one, $\hat{\Sigma}_{i} \cap \hat{\alpha}_{i}$ and hence $\Sigma_{i} \cap \alpha_{i}$ are not empty. This shows that $\left\{\Sigma_{i}\right\}$ exits $\mathcal{E}$. Under the present situation, the tameness of $\mathcal{E}$ is proved by standard arguments in hyperbolic geometry, for example see $[2 ; 6 ; 13]$ or Tameness Criteria in [5, Section 6]. However, the case when $C_{i}$ is a handlebody is exceptional. As is pointed out in the paragraph following the statement of Theorem 3 in Souto [13], we need furthermore to show that $\left[\Sigma_{i}\right] \neq 0$ in $H_{2}\left(N \backslash \delta_{1} ; \mathbf{Z}\right)$ for guaranteeing that any surface homologous to $\Sigma_{i}$ in $N \backslash C$ excises the end $\mathcal{E}$ from $N$, where $C$ is a compact core of $N$ with Int $C \supset \delta_{1}$ and $C \cap \Sigma_{i}=\varnothing$.

Suppose that a compact core of $N$ and hence any $\bar{X}_{i}$ are handlebodies. Let $J$ be a tubular neighborhood of $\delta_{1}$ in $N$ and $\widehat{J}$ a lift of $J$ to $X_{i}$ containing $\hat{\delta}_{1}$. For $i$ sufficiently large, $J \cap \Sigma_{i}$ is empty and hence $\bar{q}_{i}^{-1}(J) \cap \widehat{\Sigma}_{i} \subset \bar{q}_{i}^{-1}\left(J \cap \Sigma_{i}\right)=\varnothing$. 
The closure $K$ of the union of bounded components of $\bar{Z}_{i} \backslash\left(\widehat{\Sigma}_{i} \cup \widehat{J}\right)$ is compact. As $\bar{q}_{i}^{-1}\left(\delta_{1}\right)$ is disjoint from $\widehat{\Sigma}_{i} \cup \partial \widehat{J} \supset \partial K$, the preimage $\bar{q}_{i}^{-1}\left(\delta_{1}\right)$ contains no line components meeting $K$ nontrivially. Since $\bar{q}_{i}^{-1}\left(\delta_{1}\right) \backslash p_{i}^{-1}\left(\delta_{1}\right) \subset \partial \bar{Z}_{i}$, if $\bar{q}_{i}^{-1}\left(\delta_{1}\right) \cap$ $K$ were nonempty, then each component $\widehat{\delta}_{1}^{\prime}$ of the intersection would be a loop component of $p_{i}^{-1}\left(\delta_{1}\right)$. Since $\left(\text { inc }_{i} \circ p_{i}\right)_{*}: \pi_{1}\left(X_{i}\right) \longrightarrow \pi_{1}(N)$ is an isomorphism, $\hat{\delta}_{1}^{\prime}$ is freely homotopic in $X_{i} \subset \bar{Z}_{i}$ to $\hat{\delta}_{1}$ up to multiplicity. Then there would exist a

CAT $\left(-a^{2}\right)$-piecewise ruled annulus in $\bar{Z}_{i}$ with the geodesic boundary $\widehat{\delta}_{1} \cup \hat{\delta}_{1}^{\prime}$, which is a contradiction by the Gauss-Bonnet Theorem. It follows that $\bar{q}_{i}^{-1}\left(\delta_{1}\right) \cap K=\varnothing$, and so $[\partial \widehat{J}]+\left[\widehat{\Sigma}_{i}\right]=0$ in $H_{2}\left(\bar{Z}_{i} \backslash \bar{q}_{i}^{-1}\left(\delta_{1}\right) ; \mathbf{Z}\right)$. This shows that $\left[\Sigma_{i}\right]=-[\partial J] \neq 0$ in $H_{2}\left(N \backslash \delta_{1} ; \mathbf{Z}\right)$.

\section{References}

[1] I Agol, Tameness of hyperbolic 3-manifolds arXiv:math.DG/0405568

[2] F Bonahon, Bouts des variétés hyperboliques de dimension 3, Ann. of Math. (2) 124 (1986) 71-158 MR847953

[3] MR Bridson, A Haefliger, Metric spaces of non-positive curvature, Grundlehren series 319, Springer, Berlin (1999) MR1744486

[4] M G Brin, T L Thickstun, Open, irreducible 3-manifolds which are end 1-movable, Topology 26 (1987) 211-233 MR895574

[5] D Calegari, D Gabai, Shrinkwrapping and the taming of hyperbolic 3-manifolds, J. Amer. Math. Soc. 19 (2006) 385-446 MR2188131

[6] R D Canary, Ends of hyperbolic 3-manifolds, J. Amer. Math. Soc. 6 (1993) 1-35 MR1166330

[7] S Choi, Drilling cores of hyperbolic 3-manifolds to prove tameness, preprint (2004) arXiv: math.GT/0410381

[8] A Marden, The geometry of finitely generated kleinian groups, Ann. of Math. (2) 99 (1974) 383-462 MR0349992

[9] R Myers, End reductions, fundamental groups, and covering spaces of irreducible open 3-manifolds, Geom. Topol. 9 (2005) 971-990 MR2140996

[10] G P Scott, Compact submanifolds of 3-manifolds, J. London Math. Soc. (2) 7 (1973) 246-250 MR0326737

[11] J Simon, Compactification of covering spaces of compact 3-manifolds, Michigan Math. J. 23 (1976) 245-256 (1977) MR0431176

[12] T Soma, Covering 3-manifolds with almost compact interior, Quart. J. Math. Oxford Ser. (2) 44 (1993) 345-353 MR1240478 
[13] J Souto, A note on the tameness of hyperbolic 3-manifolds, Topology 44 (2005) 459474 MR2114957

[14] F Waldhausen, On irreducible 3-manifolds which are sufficiently large, Ann. of Math. (2) 87 (1968) 56-88 MR0224099

Department of Mathematics and Information Sciences, Tokyo Metropolitan University Minami-Ohsawa 1-1, Hachioji, Tokyo 192-0397, Japan

tsoma@center.tmu.ac.jp

Proposed: Jean-Pierre Otal

Received: 15 September 2004

Seconded: David Gabai, Tobias Colding

Revised: 26 June 2006 